\title{
Satellite Imaging of Global Urbanicity relates to Brain and Behavior in Young People
}

Gunter Schumann ( $\sim$ gunter.schumann1961@gmail.com )

Centre for Population Neuroscience and Precision Medicine (PONS) and SGDP Centre, Institute of Psychiatry, Psychology and Neuroscience, King's College London, London SE5 8AF, United Kingdom

JIAYUAN XU

Tianjin Medical University General Hospital

Xiaoxuan Liu

Tsinghua University

Alex Ing

King's College London

QIAOJUN LI

Tianjin University of Commerce

Wen Qin

Tianjin Medical University General Hospital

Lining Guo

Tianjin Medical University General Hospital

\section{Conghong Huang}

Tsinghua University https://orcid.org/0000-0002-0180-0806

Jingliang Chen

First Affiliated Hospital of Zhengzhou University

\section{Meiyun Wang}

Henan Provincial People's Hospital \& the People's Hospital of Zhengzhou University https://orcid.org/0000-0003-4454-5005

\section{Zuojun Geng}

Second Hospital of Hebei Medical University

\section{Wenzhen Zhu}

Department of Radiology, Tongji Hospital, Tongji Medical College, Huazhong University of Science \& Technology

\section{Bing Zhang}

Nanjing Drum Tower Hospital

\section{Weihua Liao}

Xiangya Hospital Central South University

\section{Shijun Qiu}

First Affiliated Hospital of Guangzhou University of Chinese Medicine

\section{Hui Zhang}

First Hospital of Shanxi Medical University Department of Radiology

\section{Xiaojun Xu}

Second Affiliated Hospital of Zhejiang University

\section{Yongqiang $\mathrm{Yu}$}

First Affiliated Hospital of Anhui Medical University

\section{Bo Gao}

Qindao University Medical College Affiliated Yantai Yuhuangding Hospital

\section{Tong Han}

Tianjin Huanhu Hospital 


\section{Guang-Bin Cui}

Tangdu Hospital, Fourth Military Medical University https://orcid.org/0000-0002-7935-9803

\section{Feng Chen}

Hainan General Hospital

Junfang Xian

Beijing Tongren Hospital

\section{Jiance Li}

First Affiliated Hospital of Wenzhou Medical University

\section{Jing Zhang}

Lanzhou University Second Hospital

\section{Xi-Nian Zuo}

BEIJING NORMAL UNIVERSITY https://orcid.org/0000-0001-9110-585X

\section{Dawei Wang}

Qilu Hospital of Shandong University

\section{Wen Shen}

Tianjin First Center Hospital

\section{Yanwei Miao}

First Affiliated Hospital of Dalian Medical University

\section{Fei Yuan}

Pingjin Hospital, Logistics University of Chinese People's Armed Police Forces

Su Lui

West China Hospital of Sichuan University

\section{Xiaochu Zhang}

University of Science and Technology of China https://orcid.org/0000-0002-7541-0130

Kai Xu

Affiliated Hospital of Xuzhou Medical College

\section{Long Jiang Zhang}

Jinling Hospital, Medical School of Nanjing University https://orcid.org/0000-0002-6664-7224

\section{Zhaoxiang Ye}

Tianjin Medical University Cancer Institute and Hospital

\section{Tobias Banaschewski}

Central Institute of Mental Health, University of Heidelberg https://orcid.org/0000-0003-4595-1144

\section{Gareth Barker}

Institute of Psychiatry, Psychology \& Neuroscience, Kings College London https://orcid.org/0000-0002-5214-7421

\section{Arun Bokde}

Trinity College Dublin https://orcid.org/0000-0003-0114-4914

\section{Erin Quinlan}

King's College London https://orcid.org/0000-0003-2927-1632

\section{Herta Flor}

Central Institute of Mental Health Medical Faculty Mannheim Heidelberg University

\section{Antoine Grigis}

University of Paris-Saclay

\section{Hugh Garavan}

University of Vermont

\section{Penny Gowland}

University of Nottingham https://orcid.org/0000-0002-4900-4817

\section{Andreas Heinz}

Charité Universitätsmedizin, Berlin https://orcid.org/0000-0001-5405-9065 
Rüdiger Brühl

Physikalisch-Technische Bundesanstalt

Jean-Luc Martinot

Paris Descartes University https://orcid.org/0000-0002-0136-0388

\section{Eric Artiges}

INSERM/Université Paris-Saclay https://orcid.org/0000-0003-4461-7646

Frauke Nees

Central Institute of Mental Health https://orcid.org/0000-0002-7796-8234

\section{Dimitri Papadopoulos Orfanos}

CEA https://orcid.org/0000-0002-1242-8990

\section{Herve Lemaitre}

University of Paris-Saclay

\section{Tomas Paus}

University of Toronto https://orcid.org/0000-0003-1495-9338

\section{Luise Poustka}

Universitätsmedizin Göttingen

\section{Sarah Hohmann}

Heidelberg University

Juliane Fröhner

TU Dresden https://orcid.org/0000-0002-8493-6396

\section{Michael Smolka}

Technische Universität Dresden https://orcid.org/0000-0001-5398-5569

\section{Henrik Walter}

Charité - Universitätsmedizin Berlin, corporate member of Freie Universität Berlin, Humboldt-Universität zu Berlin https://orcid.org/0000-0002-9403-6121

\section{Robert Whelan}

Trinity College Dublin

\section{Ran Goldblatt}

New Light Technologies Inc

\section{Kevin Patrick}

University of California San Diego Department of Family and Preventive Medicine

\section{Vince Calhoun}

Emory University

\section{Mulin Lijun}

Tianjin Medical University

\section{Meng Liang}

Tianjin Medical University General Hospital

\section{Peng Gong}

Tsinghua University https://orcid.org/0000-0003-1513-3765

\section{Edward Barker}

Institute of Psychiatry, King's College London https://orcid.org/0000-0002-9914-8958

\section{Nicholas Clinton}

Google, Inc., 1600 Amphitheatre Parkway, Mountain View, CA 94043, USA

\section{Le Yu}

Tianjin Medical University General Hospital

\section{Chunshui Yu}

Tianjin Medical University General Hospital

\section{Qiang Luo}


Fudan University https://orcid.org/0000-0002-0426-6039

\section{Huaigui Liu}

Tianjin Medical University General Hospital

\section{Congying Chu}

Centre for Population Neuroscience and Stratified Medicine (PONS) and MRC-SGDP Centre, Institute of Psychiatry, Psychology \& Neuroscience

\section{Feng Liu}

Tianjin Medical University General Hospital

\section{IMAGEN Consortium}

Centre for Population Neuroscience and Precision Medicine (PONS) and SGDP Centre, Institute of Psychiatry, Psychology and Neuroscience, King's College London, London SE5 8AF, United Kingdom

\section{CHIMGEN Consortium}

Tianjin Medical University General Hospital

Article

Keywords: urbanicity, environmental challenge, mental health, brain, behavior, young people

Posted Date: April 1st, 2021

DOI: https://doi.org/10.21203/rs.3.rs-155206/v1

License: (c) (i) This work is licensed under a Creative Commons Attribution 4.0 International License. Read Full License

Version of Record: A version of this preprint was published at Nature Human Behaviour on October 28th, 2021. See the published version at https://doi.org/10.1038/s41562-021-01204-7. 


\section{Abstract}

Urbanicity is a growing environmental challenge for mental-health. While the impact of urban life on brain and behavior might be distinct in different sociocultural conditions and geographies, there might exist features shared between regions. To investigate correlations of urbanicity with brain structure and function, neuropsychology and mental illness symptoms in young people from China and Europe, we developed a remote-sensing satellite-measure termed 'UrbanSat' quantifying populationdensity, a general measure of urbanicity. UrbanSat is correlated with brain volume, surface area and brain-network-connectivity in the medial prefrontal cortex and cerebellum, which mediate its effect on perspective-taking and depression- symptoms. Susceptibility to high population-density is greatest during childhood for the cerebellum and from childhood to adolescence for the prefrontal cortex. As UrbanSat can be generalized to different geographies, it will enable assessing the impact of urbanicity on mental illness and resilience globally, especially in young people where prevention and early interventions are most effective.

\section{Introduction}

Mental disorders account for $28 \%$ of disease burden among non-communicable diseases ${ }^{1}$. Environmental factors account for up to $50 \%$ of the attributable risk for mental disorders ${ }^{2}$. The environmental measures investigated in mental-health research include not only individual life events ${ }^{3}$, such as trauma, abuse, neglect, or psychosocial stress, but also, albeit to a lesser extent, individual physical environments ${ }^{4}$.

Urbanicity, the living conditions particular to urban areas, is among the most important environmental challenges globally 5 . While the physical environments are hallmarks of a city, urbanicity also includes the social environment and access to health and social services ${ }^{5}$. The physical, social and service dimensions of urbanicity form a complex relation with each other that has hitherto prevented the development of a unifying concept and measurement of urbanicity ${ }^{5}$.

In 1950 , less than $30 \%$ of the world's population lived in urban areas, but this number has increased to presently $55 \%$ and is expected to rise to $68 \%$ in $2050^{6}$. While Europe is among the most stable urbanized regions, Asia is home to $54 \%$ of the world's urban population and subject to massive demographic changes: for example, by 2050 China will have added 255 million urban dwellers ${ }^{7}$. The increasing global urban population emphasizes the importance of investigating how the living conditions particular to urban areas affect human brain and behavior.

We were interested to investigate the relation of urbanicity with brain and behavior in different sociocultural conditions and geographies and further to identify possible susceptibility periods across the life span in young people, enabling targeted preventions when the developing brain may benefit most from environmental modification. Whereas there might be distinct influences of increased population-density in urban settings in different sociocultural conditions and geographies, there are likely to exist common associations with brain and behavior shared in different areas of the globe.

Several studies have focused on the relation of individual physical environments linked to urban living with brain and behavior, such as green space, air and noise pollution ${ }^{4,8}$. However, a more general measure of urbanicity that can objectively assess urban environment with high spatiotemporal resolution and coverage is still lacking. Such a general measure is important, as it registers the overall and susceptibility-period effects of urbanicity on brain and behavior, and may in a subsequent step enable the identification and ranking of the individual features of the physical, social and service environment, and their interactions, that contribute most to the observed relation.

Traditionally, the characterization to urbanicity was carried out using census data, which are ascertained infrequently in different ways and at different times in different countries ${ }^{5}$. Thus, census data are less useful for comparing urbanicity across different countries. More recently, the Global Human Settlement Layer (GHSL) has provided globally standardised human settlements, including urbanisation and urbanicity ${ }^{9}$. GHSL data, however, are only available at large and infrequent intervals, namely 1975 , 1990, 2000 and $2015^{9}$. 
To facilitate global comparative analyses of the overall effects of urbanicity on brain and behavior and to identify potential susceptibility-periods, dense quantitative and longitudinal environmental measures that can be obtained from different geographies are required. Remotely-sensed satellite-data provide globally standardized quantitative environmental measures enabling the tracing of environmental features going back nearly 50 years ${ }^{10}$. Population-density is a well-established and quantifiable general measure of urbanicity, frequently applied for neighbourhood classification and used around the globe ${ }^{9}$. Here, we aimed to use population-density as a general measure of urbanicity to investigate if the urban environment is correlated with brain and behavior, and if these correlations are comparable in China and Europe. Specifically, we developed a satellite-based measure of population-density termed 'UrbanSat', and applied UrbanSat in China and Europe to investigate the relation of population-density, as a proxy of urbanicity, with brain structure, function and behavior in two neuroimaging datasets of young people: as exploration dataset, we used the Chinese CHIMGEN cohort (www.chimgen.tmu.edu.cn) ${ }^{11}$ and as replication dataset, we used the European longitudinal IMAGEN-cohort (www.imagen-europe.com) ${ }^{12}$. While we did not have any a priori hypotheses, we were interested in investigating if: (i) UrbanSat is associated with brain structure, function and behavior; (ii) Brain features associated with UrbanSat mediate the association between UrbanSat and behavior; (iii) Correlations of UrbanSat with brain and behavior are similar in Chinese and Europeans; Furthermore, we were interested in (iv) identifying susceptibilityperiods for the effects of UrbanSat during child and adolescent development on brain and behavior. A schematic summary is shown in Fig. 1.

\section{Results}

\section{Demographics}

We recruited young-adult participants with lifetime-residential geographies from CHIMGEN $(n=3306)$ and IMAGEN second follow-up (FU2) (n=561). Detailed inclusion and exclusion criteria are presented in Supplementary Tables 1 and 2. Demographics of samples used in statistical analyses and sample attrition are described in Supplementary Table 3 and Supplementary Figs.1-2. Demographic comparisons between the analysed sample and total sample are shown in Supplementary Table 4. Demographic variables showing significant differences between the analysed sample and excluded sample were adjusted during analyses (Online Methods and Supplementary Tables 5-6).

\section{UrbanSat: a satellite-based measure of urbanicity}

To develop a satellite-based measure of urbanicity, we selected information from nine types of satellite registrations relevant for detecting and characterising urban settlements, including night-time light (NL), normalized difference built-up index (NDBI), normalized difference water index (NDWI), normalized difference vegetation index (NDVI), and five measures derived from land cover mapping (Built-up\%, cropland\%, grassland\%, forest\% and water body\%) (Online and Supplementary Methods, Supplementary Table 7). After imputing the nine annual satellite registrations for the participants with missing values using Bayesian data augmentation (Supplementary Table 8 and Online Methods), we carried out a ten-fold cross validation stratified by spatiotemporality to optimize the confirmatory factor analysis (CFA) models, and to predict annual UrbanSat scores of each participant from birth to the age of recruitment (Online Methods). UrbanSat was generated by the optimized CFA model consisting of NL, built-up\%, cropland\% and NDVI, which best captured variation of urban features while maximizing goodnessof-fit. UrbanSat in CHIMGEN (Supplementary Tables 9-10) and IMAGEN-FU2 (Supplementary Tables 11-12) had a Tucker-LewisIndex (TLI) and comparative-fit-index (CFI) $>0.95$, root mean-square-error of approximation (RMSEA) $<0.06$ and standard root mean-square-residual (SRMR) $<0.08$, indicating excellent model fit (Online Methods). UrbanSat was robust across time and geographies, as validated by its correlations with ground-level population-density from GHSL-POP ${ }^{9}$ for China and Europe for the years 1990, 2000 and 2015 (Fig.2). Histograms of the distribution of UrbanSat score in each center are shown in Supplementary Fig.3. UrbanSat showed higher correlations with population-density in different residential categories (rural, town, city and overall), countries (Asia and Europe) and years (1990, 2000 and 2015) than any individual satellite-measures (Fig. 2).

\section{Correlations of UrbanSat with brain structure}


Voxel-wise multiple-regression of mean UrbanSat before age 18 with brain gray-matter-volume (GMV) was performed in CHIMGEN ( $n=2176)$. We controlled for age in all analyses, thus accounting for the older and wider age spread in CHIMGEN (age: $23.54 \pm 2.33$ years) compared to IMAGEN (age: $18.89 \pm 0.66$ years). We also controlled throughout for gender, education, site, body-mass-index (BMI), genetic population-stratification and socioeconomic status (SES) (Supplementary Tables 13-14). Total intracranial volume was controlled in all imaging analyses, except for the analyses of cortical thickness (CT) and surface area (SA), where mean CT and total SA were controlled, respectively. Parental history of mental illness was an exclusion criterion for CHIMGEN and controlled for in IMAGEN. Uncorrected statistical maps of the association of UrbanSat with brain GMV in CHIMGEN adjusting for confounding covariates under parametric testing and non-parametric permutation testing are shown in Fig.3a and Supplementary Fig. 4. We found negative correlation of UrbanSat with medial prefrontal cortex (mPFC) volume (peak MNI-coordinate: $x=-7.5, y=30, z=45 ; 676$ voxels; peak $t$-value=-6.42; Fig.3b) and a positive correlation with cerebellar volume (peak MNI-coordinate: $\mathrm{x}=10.5, \mathrm{y}=-51, \mathrm{z}=-18 ; 978$ voxels; peak $t$-value $=6.22$; Fig.3b) (Parametric testing $P_{\mathrm{c}}<0.05$, family-wise error (FWE) corrected for voxel numbers, imaging modalities and data categories; see Online Methods). We confirmed the results with non-parametric permutation-testing (TFCE-FWE, $P c<0.05$, Supplementary Methods and Supplementary Fig.4). Potential imputation-bias was ruled-out by sensitivity-analyses in 1491 participants with complete satellite and neuroimaging data (Supplementary Fig.5 and Supplementary Results). Uncorrected and adjusted vertex-wise correlation maps of UrbanSat with whole-brain CT and SA are shown in Supplementary Fig.6 ( $n=2164)$. The mPFC-region of interest (ROI) from GMV-analyses was projected onto fsaverage surface of Freesurfer v5.3.0 (Supplementary Fig.7). UrbanSat was correlated with mean SA ( $r h o=-0.07$, $P=8.12 \times 10^{-4}$ ) but not mean CT ( $r h o=-0.02, P=0.28$ ) of the mPFC cluster (Fig.3b and Supplementary Table 15). Using voxel-wise multiple regression of individual satellite- measures with GMV, we found significant correlation with mPFC-GMV being driven by $\mathrm{NL}$ and built-up\%, and correlation with cerebellar-GMV being driven by NL and cropland\%. We found no correlation of NDVI with either mPFC- or cerebellar-GMV (Supplementary Fig.8). We observed similar results in GHSL ground-level population-density data, thus validating the relation of UrbanSat and GMV (Supplementary Fig.8).

In IMAGEN-FU2 ( $\mathrm{n}=415)$, we replicated CHIMGEN findings. The uncorrected statistical correlation map of UrbanSat with brain GMV in CHIMGEN showed significant spatial correlation $(r=0.40, P<0.001)$ with that of IMAGEN-FU2 (Supplementary Fig.9). UrbanSat was correlated with GMVs of the mPFC ( $\left.r h o=-0.20, P=4.49 \times 10^{-5}\right)$ and cerebellum $(r h o=0.11, P=0.03)$, and SA of the $\operatorname{mPFC}\left(r h o=-0.15, P=3.01 \times 10^{-3}\right)$, but not CT of the mPFC ( $\left.r h o=-0.03, P=0.58\right)$ (Fig.3b and Supplementary Table 15). In voxel-wise analyses, we validated negative correlation of UrbanSat with mPFC volume and positive correlation with cerebellar volume (Supplementary Fig.10), both driven by NL and built-up\% (Supplementary Fig.11).

To exclude possible scanner and site effects, we performed separate analyses for each acquisition-site of CHIMGEN and IMAGEN-FU2, carrying out a meta-analysis with an inverse variance-weighted random-effects model (Online and Supplementary Methods). UrbanSat remained significantly negatively correlated with mPFC-GMV and SA, and positively correlated with the cerebellar-GMV (Supplementary Fig.12 and Supplementary Table 16). Heterogeneity of effect sizes was from low to moderate for all regions ( $P^{\text {rrange: }}$ 0.19\%-41.35\%) (Supplementary Table 16). Thus, the observed correlation between UrbanSat and brain structure is robust across geographies and socio-cultural conditions.

We applied distributed lag models (DLMs) to identify susceptibility-periods of lifetime UrbanSat on GMV and SA (Online Methods). We observed a negative association of UrbanSat with mPFC-GMV from age 4 to 15 (Fig.3c) and SA from age 5 to 7 (Fig.3c), indicating a susceptibility-period during childhood and adolescence, driven by NL, built-up\%, cropland\% and NDVI (Supplementary Fig.13). Correlation of UrbanSat with cerebellar GMV was significant from age 1 to 10 years, indicating a susceptibility-period during childhood (Fig.3c), driven by NL, built-up\% and cropland\% (Supplementary Fig.13).

To investigate the relation between UrbanSat and brain development, we used the longitudinal IMAGEN dataset to calculate volumetric $(n=340)$ and SA/CT change-rate/year $(n=325)$ between baseline $(B L)$ at 14 and FU2 at 19 years (IMAGEN BL-FU2). Consistent with the susceptibility periods identified, UrbanSat was significantly correlated with brain volumetric development in the mPFC-ROI ( $\left.r h o=0.17, P=2.10 \times 10^{-3}\right)$, but not with the cerebellum-ROI $(r h o=0.02, P=0.70)$. This correlation was driven by $\mathrm{mPFC}$ SA changes $\left(r h o=0.24, P=1.31 \times 10^{-5}\right)$, not by CT changes ( $\left.r h o=-0.01, P=0.93\right)$ (Supplementary Table 15). 
To measure the relation between age of migration and brain structure, we split CHIMGEN participants into who migrated to the city before age 14 years $(n=229$, mean-age at migration $=8.24 \pm 4.86$ years), after age $14(n=1385$, mean-age at migration=17.17 \pm 2.68 years), and life-long city-dwellers $(n=562)$ (Fig.3d). We found that participants born in the city or early migrants showed smaller mPFC-GMV $(P=0.040)$ and SA $\left(P=7.28 \times 10^{-9}\right)$ as well as greater cerebellar-GMV $\left(P=5.00 \times 10^{-5}\right)$ than those with later exposure (Fig.3e and Supplementary Table 17).

\section{No correlation of UrbanSat with white-matter microstructure}

Using tract-based spatial statistics (TBSS) analysis of diffusion-tensor imaging (DTI) data, we did not find significant correlation of UrbanSat with brain fractional anisotropy (FA) in either CHIMGEN or IMAGEN-FU2 (TFCE-FWE $P_{\mathrm{c}}<0.05$ ).

\section{Correlations of UrbanSat with resting-state functional network connectivity}

Using group-independent-component-analysis (GICA) of estimated 30 independent components (Supplementary Methods), we identified 17 resting-state networks (RSNs) related to cognitive and sensory-motor processes ${ }^{13}$ in both CHIMGEN and IMAGEN ( $n=2156$ ) (Supplementary Fig.14). For each RSN, we tested the relation between mean UrbanSat and within-network functional connectivity (WNFC). A voxel-wise multiple-regression analysis controlling for all confounders revealed a negative correlation of UrbanSat with WNFC in the mPFC of the anterior default-mode-network (aDMN) (peak MNI-coordinate: $x=-3.5, y=69, z=0 ; 142$ voxels; peak $t$-value=-6.96), and positive correlations in the cerebellar vermis of the cerebellar-network (CN) (peak MNIcoordinate: $x=3, y=-72, z=-9 ; 122$ voxels; peak $t$-value $=7.21$ ), in the left lingual gyrus (LG) of the medial visual-network (mVN) (peak MNI-coordinate: $x=-12, y=-90, z=3.5 ; 143$ voxels; peak $t$-value $=6.97$ ) and in the left $L G$ of the lateral visual-network (IVN) (peak MNl-coordinate: $\mathrm{x}=-24, \mathrm{y}=-81, \mathrm{z}=-12$; 141 voxels; peak $t$-value=6.97) (FWE $P_{\mathrm{c}}<0.05$, additionally corrected for $17 \mathrm{RSNs}$, Online Methods) (Fig. 4a). Voxel-based correlations of individual satellite-measures with WNFCs of each RSN are shown in Supplementary Fig.15. The correlations of UrbanSat with WNFCs in CHIMGEN were replicated in ROI-based analyses in IMAGENFU2 (n=351) (aDMN: $r h o=-0.18, P=7.20 \times 10^{-4} ; \mathrm{CN}$ : $r h o=0.21, P=1.41 \times 10^{-4} ; \mathrm{mVN}$ : $r h o=0.24, P=1.05 \times 10^{-5}$; IVN: $r h o=0.19, P=3.97 \times 10^{-}$

${ }^{4}$ ) (Fig.4b and Supplementary Table 15). Only the aDMN and CN results were replicated in voxel-wise analyses in IMAGEN-FU2 (Supplementary Fig.16).

In 136 between-network functional connectivity (BNFC), UrbanSat was correlated with 49 BNFCs in CHIMGEN $\left(P_{c}<0.05,10,000\right.$ permutations, see Online Methods) (Fig.4e), four of which were replicated in IMAGEN-FU2 (Fig.4f). These four BNFCs (aDMN-CN, aDMN-ECN, aDMN-rFPN and rFPN-IFPN) connect five brain functional-networks (aDMN; CN; executive-control-network (ECN); rFPN/IFPN, right or left frontoparietal-network (FPN)), implicated in self-referential thoughts ${ }^{14}$ and executive control ${ }^{15}$.

The correlations of UrbanSat with WNFCs and BNFCs were stable in a meta-analysis of all CHIMGEN and IMAGEN sites (Supplementary Fig.12 and Supplementary Table 16). Brain localization (Fig.4b and Fig.4f) and susceptibility- periods (Fig.4d and Fig.4h) of WNFCs and BNFCs in CHIMGEN and IMAGEN were consistent with those observed for brain structure (Supplementary Fig.15-17), except for non-significant during adolescence. The WNFCs and BNFCs changes between 14 and 19 years in IMAGEN were correlated with UrbanSat (Fig.4b and Fig.4f and Supplementary Table 15 and Supplementary Results). In CHIMGEN, WNFCs and BNFCs were correlated with age of migration to the city (Fig. 4c and Fig.4g and Supplementary Results).

\section{Correlations of UrbanSat with behavior}

We investigated whether UrbanSat is related to measures of cognition and mental- health, i.e. depression and anxiety. In CHIMGEN ( $n=2148)$, the social-cognition measure 'perspective-taking', perceiving a situation from an alternative point of view ${ }^{16}$, was positively correlated with UrbanSat (reaction-time for perspective-taking: $r h o=-0.14, P_{\mathrm{c}}<0.05$, Bonferroni-corrected for datacategories and 21 behavioral assessments, see Online Methods) and replicated in IMAGEN-FU2 $\left(r h o=0.14, P_{\mathrm{c}}<0.05\right)($ Table 1$)$. A negative correlation between UrbanSat and reaction-time for perspective-taking performance was observed from 12 to 22 years in CHIMGEN (Fig.5a).

UrbanSat was correlated with depression-symptoms assessed by Beck-Depression-Inventory (BDI) in CHIMGEN ( $n=2170)$ $\left(\right.$ rho $\left.=0.14, P_{c}<0.05\right)$ (Table 1) with a susceptibility-period from 3 to 12 years (Fig. 5a). As BDI was not available in IMAGEN, we 
validated this association using an instrument measuring core features of depression, the Ruminating-Scale-Questionnaire (RSQ) ( $\left.r h o=0.14, P_{\mathrm{c}}<0.05\right)$ (Table 1 and Supplementary Methods).

In CHIMGEN and IMAGEN, increased NL and built-up\%, decreased NDVI and cropland\% were significantly correlated with enhanced perspective-taking performance and increased depression-symptoms (Table 1). The susceptibility-periods for individual satellite-measures were similar to UrbanSat in CHIMGEN (Supplementary Fig.18). Although most correlations of UrbanSat with brain and behaviour were consistent between males and females, some correlations, especially with brain development in IMAGEN BL-FU2, were sex-specific (Supplementary Tables 19-20).

\section{Multiple mediation in UrbanSat-brain-behavior}

We applied multiple-mediation analysis to investigate if the significant brain imaging measures mediate correlations of UrbanSat with perspective-taking and depression-symptoms in CHIMGEN and IMAGEN-FU2 (Online Methods). In CHIMGEN, $19.32 \%$ of the correlation between UrbanSat and reaction-time for perspective-taking was mediated by brain, namely mPFC-GMV (2.55\%), the cerebellar-GMV (2.94\%), WNFCs in aDMN (2.89\%) and CN (3.21\%), as well as by the BNFCs of the aDMN-CN (2.27\%), aDMN-ECN (4.54\%) and aDMN-rFPN (4.14\%) (Fig. 5b). Mediation was replicated in IMAGEN-FU2, with the association of UrbanSat with perspective-taking being mediated by the mPFC-GMV (1.47\%), WNFCs in the aDMN (1.96\%) and CN $(0.89 \%)$ as well as BNFCs of the aDMN-CN (1.45\%) and aDMN-rFPN (1.22\%) (Fig.5b). There was no mediation of the cerebellar GMV in IMAGEN-FU2 (Supplementary Table 21).

In CHIMGEN, $20.32 \%$ of the correlation between UrbanSat and BDI was mediated by brain, namely mPFC-GMV (4.81\%) and SA (1.80\%), cerebellar-GMV (6.88\%), WNFCs in aDMN (2.45\%) and mVN (2.18\%), BNFC of aDMN-ECN (4.04\%) (Fig.5c). In IMAGENFU2 the correlation between UrbanSat and rumination was mediated by mPFC GMV (1.93\%), WNFC in aDMN (0.96\%) and BNFC of the aDMN-ECN (1.62\%) (Fig.5c), but not by the cerebellar-GMV (Supplementary Table 21).

\section{Discussion}

Using a remote-sensing satellite-measure, 'UrbanSat', we characterized the relation of population-density, a proxy of urbanicity, with brain structure, function and behavior during childhood and adolescence in large datasets in China and Europe. We provide converging evidence for association of UrbanSat during childhood and adolescence with GMV and SA of the mPFC and aDMN, but not with CT and FA. The MPFC and aDMN mediate the correlation between UrbanSat and improved perspective-taking and increased depression-symptoms. We also found positive correlations of UrbanSat during childhood with cerebellar volume, which mediated the association with perspective-taking and depression-symptoms. We are extending previous observations reporting an association of depression-symptoms with urban settings ${ }^{17}$, by demonstrating the stability of this observation in different geographical and sociocultural regions, and by discovering possible underlying brain mechanisms and susceptibilityperiods during childhood and adolescent development.

Our results suggest urban living has both beneficial and adverse correlations with health: enhanced social cognition (perspective-taking) and increased depression-symptoms, in contrast to previous studies, which mainly reported adverse aspects of urbanicity ${ }^{18}$. The mPFC, the core brain area of the aDMN, has been implicated in a variety of social-cognition and affective functions commonly compromised in psychiatric disorders ${ }^{19}$. The susceptibility of mPFC to urban environment is supported by the greater sensitivity of the MPFC, to urbanicity-related risk-factors, including chronic stress ${ }^{20}$ and air-pollution $^{21}$. While our findings are consistent with reports of an association between urbanicity and mPFC in smaller European samples ${ }^{22}$, they differ from these studies as we found associations with GMV and SA rather than CT, and an absence of sex specificity.

We found a positive correlation of UrbanSat with cerebellar volume, a mediator for the association of UrbanSat with perspectivetaking and depression-symptoms. The functional network connectivity of the cerebellum also mediates the association of UrbanSat with perspective-taking. Cerebellar lesions cause the 'Cerebellar-Cognitive-Affective-Syndrome', characterized by impairments in executive-function and memory, as well as affect ${ }^{23}$. Animal studies extend these findings to stress-dependent depressive affect ${ }^{24}$ and impairment in social behavior ${ }^{25}$. It is tempting to speculate that these pathways may connect to brain 
regions involved in perspective-taking and depression-symptoms ${ }^{26}$. Imaging features related to cerebellum showed susceptibility-periods to high population-density at the age of 1-10 years, during which cerebellum and cortex are increasing in volume ${ }^{27-29}$.

While previous studies focused on the effect of mean exposure to urban-living on brain and mental-health ${ }^{30}$, we identified neurodevelopmental periods with increased susceptibility to urban-living. Consistent with observations of susceptibility-periods of non-affective psychosis to residential mobility during childhood and adolescence ${ }^{31}$, we found that structure and function of the mPFC, as well as depression-symptoms have pronounced susceptibility to high population-density during childhood and adolescence, a period more sensitive to social stress ${ }^{32}$. The correlation of UrbanSat with MPFC-GMV, and change rate, was driven by SA rather than CT, indicating that SA may be more sensitive to environmental factors than CT. Perspective-taking was more sensitive to high population-density during adolescence and young adulthood, implying a time-window for neurobehavioural interventions targeting social-cognition.

Our results are suggestive of a cumulative effect of urbanicity on brain and behavior, whereby participants born or migrating to the city at an earlier age had more pronounced effects than those who become city dwellers later. Given that CHIMGEN participants were students who moved to cities for their studies, we do not have any data on people who after spending some years in the city moved back to the country side. We also do not have data to distinguish possible short but extreme exposure to urban life, in utero or during susceptibility periods from a moderate continuous exposure.

We found several shared effects of high population-density in urban settings on brain structure and function as well as on social-cognition and mental-health in both CHIMGEN and IMAGEN, indicating their generalization to other sociocultural conditions and geographies. The effect of urban living on brain development during adolescence was confirmed by exploring the correlation of UrbanSat with brain structural and functional changes from age 14 to 19 in IMAGEN. Taking into account normative references ${ }^{27-29}$, our observations are consistent with an accelerated development in densely populated urban areas of cerebellum during childhood and mPFC during childhood and adolescence. We also found inconsistent results between CHIMGEN and IMAGEN: only 4/49 BNFCs correlating with UrbanSat in CHIMGEN were replicated in IMAGEN. The more extensive effects of urban living on BNFCs in CHIMGEN may reflect the more drastic changes in urbanization in China compared to Europe $^{6}$, but may also relate to confounding factors beyond the covariates controlled in our study ${ }^{33}$.

UrbanSat was correlated with GMV, SA and functional connectivity, but not with FA and CT, indicating different sensitivities of brain properties to residential environments. UrbanSat showed positive correlation with cerebellar volume, negative correlation with $\mathrm{mPFC}$ volume, but non-significant correlation with volumes of other regions, suggesting different spatial sensitivities to residential environments; the cerebellum was sensitive to urban residential environments during childhood, but the mPFC sensitive during both childhood and adolescence, indicating different temporal sensitivities to residential environments. This framework of different spatial and temporal sensitivities to urban residential environments may help to understand the association of urban living with brain and mental-health.

High population-density a general measure of urbanicity, can cause increased social stress and air pollution, both of which affect brain structure in young people $\mathrm{e}^{34,35}$. A recent study has observed an association of urbanicity with brain activity in regions linked to social stress processing ${ }^{30}$. Such brain changes may mediate the well-established impact of urbanicity on mental-health, including on mood disorders ${ }^{36}$ and social-cognition ${ }^{37}$. Stress in childhood can accelerate brain development and lead to faster maturation of certain brain regions during adolescence, including cerebellum and the mPFC ${ }^{38}$. Faster brain maturation results in enhanced cognitive development ${ }^{39}$ and may account in part for the positive correlation of urbanicity and perspective-taking observed in our study. However, faster maturation of the mPFC and cerebellum may come at a cost of decreased plasticity, including of fear extinction mechanisms (mPFC), which may contribute to increased vulnerability for anxiety and depression ${ }^{40}$. Air pollution induces neuroinflammation in the brain, leading to the damage and loss of neural tissue in the prefrontal cortex ${ }^{35}$ and may provoke depression-symptoms ${ }^{41}$. Thus, urban upbringing may cause affective and anxiety symptoms by way of both, increased social stress and pollution. 
Remotely-sense satellite data play a critical role in monitoring the Earth's surface to track environmental conditions that are intimately related to human health ${ }^{10}$. Satellite data are applied to map urbanization, poverty, climate change and pollution, as well as spread of infectious disease ${ }^{10}$. Our study extends the application of remote-sensing satellite data and provides a method to characterize and monitor spatial and temporal patterns of risk for mental disorders. In the optimized CFA models, the four satellite- measures contributing to UrbanSat showed different factor-loadings. Night-time-light with the highest factorloading can capture the physical environmental features of urbanicity, such as patterns of human settlements ${ }^{42}$, urban expansion $^{43}$ and population counts ${ }^{44}$, as well as information about social-environmental features of urbanicity, such as economic activity ${ }^{45}$. Built-up\% and cropland\% with medium factor-loadings mainly reflect physical-environmental features of urbanicity. NDVI with the smallest factor-loading measures residential greenness and has been used extensively to record distribution of green spaces in urban settings ${ }^{46}$. UrbanSat mainly reflects the physical- environmental features and indicates its social-environmental features of urbanicity only indirectly.

For privacy reasons, our satellite-measures were obfuscated to a spatial resolution of one kilometer, preventing the capture of important aspects of urban life, such as daily mobility paths. Future studies will investigate the integrated effect of urban physical and social environment, their interaction with genetics and relation to brain and behavior. This study is not epidemiological but neurobiological, aiming to identify brain mechanisms by which urbanicity influences behavior. How representative the identified mechanisms are among the general population is a different task for future epidemiological studies.

Our findings were made possible due to recent advancements in remote-sensing satellite technologies which were leveraged to measure the relation of urbanicity with brain and behavior. We were able to (1) apply a general measure of urbanicity, population-density, which is not dependent on census definitions of urban areas that might be conflated by densely populated rural areas, or sparsely populated areas within urban settlements, or may vary between nations ${ }^{5}$; (2) obtain a high spatial and temporal resolution ${ }^{10}$; and (3) use a measure applicable anywhere on earth from 1970 s to the present day. Thus, UrbanSat provides a unique opportunity to identify the cumulative effects and susceptibility-periods of urbanicity on brain and behavior. However, we note that UrbanSat cannot unravel environmental pathways and their interactions that cause the aversive effects of urban living. This is a task for subsequent studies with access to sufficient ground level data for comprehensive characterization of causal environmental pathways that underlie the observed correlations.

In the current work, we have provided proof of principle establishing the use of satellite-data to inform the relation between urban environment, brain and behavior. As our approach can be extended and generalized to other geographies and is easy to implement even in the absence of detailed or directly comparable ground level data, it may be relevant for public health, policy and urban planning globally.

\section{Table 1. Correlations of UrbanSat and individual satellite-measures with behavior in CHIMGEN and IMAGEN}




\begin{tabular}{|c|c|c|c|c|c|c|}
\hline Item & Statistics ${ }^{\#}$ & UrbanSat* & Night-time light & Built-up\% & Cropland\% & NDVI \\
\hline \multicolumn{7}{|l|}{ CHIMGEN } \\
\hline \multicolumn{7}{|c|}{ PT and Ag $(n=2148)$} \\
\hline $\mathrm{ACC}_{\mathrm{pt}}$ & $\begin{array}{l}0.21 \\
(0.38)\end{array}$ & $0.14(-0.03)$ & $0.05(-0.04)$ & $0.42(-0.02)$ & $0.13(0.03)$ & $0.02(0.05)$ \\
\hline ACC $_{\text {agency }}$ & $\begin{array}{l}1.00 \times 10^{-9} \\
(0.21)\end{array}$ & $0.75(-0.01)$ & $0.57(-0.007)$ & $0.30(-0.02)$ & $0.77(-0.006)$ & $0.87(0.004)$ \\
\hline $\mathrm{RT}_{\mathrm{pt}}(\mathrm{ms})$ & $\begin{array}{l}1160.67 \\
(740.39)\end{array}$ & $2.26 \times 10^{-11}(-0.14)$ & $6.09 \times 10^{-11}(-0.14)$ & $3.53 \times 10^{-9}(-0.13)$ & $2.35 \times 10^{-10}(0.14)$ & $4.62 \times 10^{-10}(0.14)$ \\
\hline $\mathrm{RT}_{\mathrm{agency}}(\mathrm{ms})$ & $\begin{array}{l}-7.44 \\
(363.22)\end{array}$ & $0.78(0.01)$ & $0.34(0.02)$ & $0.60(0.01)$ & $0.88(-0.003)$ & $0.13(-0.03)$ \\
\hline
\end{tabular}

Mental-health ( $\mathrm{n}=2170)$

\begin{tabular}{|c|c|c|c|c|c|c|}
\hline BDI & $\begin{array}{l}2.00 \\
(5.00)\end{array}$ & $3.10 \times 10^{-11}(0.14)$ & $2.01 \times 10^{-13}(0.16)$ & $5.18 \times 10^{-7}(0.11)$ & $1.49 \times 10^{-10}(-0.14)$ & $5.90 \times 10^{-6}(-0.10)$ \\
\hline SA & $\begin{array}{l}30.00 \\
(9.00)\end{array}$ & $0.62(0.01)$ & $0.56(0.01)$ & $0.75(-0.007)$ & $0.46(-0.02)$ & $0.80(-0.01)$ \\
\hline TA & $\begin{array}{l}33.00 \\
(9.00)\end{array}$ & $0.28(0.02)$ & $0.07(0.04)$ & $0.47(0.04)$ & $0.27(-0.02)$ & $0.39(-0.02)$ \\
\hline
\end{tabular}

\section{IMAGEN FU2}

PT
IRI $(n=342) \quad 19.00$
$9.11 \times 10^{-3}(0.14) \quad 0.74(0.02)$
$9.21 \times 10^{-3}(0.14) \quad 0.08(-0.10)$
$0.81(0.01)$

(5.00)

\section{Mental-health}

\begin{tabular}{|c|c|c|c|c|c|c|}
\hline RSQ ( $n=346)$ & $\begin{array}{l}35.00 \\
(15.00)\end{array}$ & $9.02 \times 10^{-3}(0.14)$ & $0.45(0.04)$ & $8.58 \times 10^{-3}(0.14)$ & $0.90(0.007)$ & $0.56(0.03)$ \\
\hline $\begin{array}{l}\text { DAWBA-GA } \\
(\mathrm{n}=447)\end{array}$ & $355 / 92$ & $0.94(-0.01)$ & $0.97(-0.003)$ & $0.84(0.09)$ & $0.18(-0.65)$ & $0.61\left(-2.57 \times 10^{-4}\right)$ \\
\hline \multicolumn{7}{|l|}{$(\mathrm{Y} / \mathrm{N})$} \\
\hline $\begin{array}{l}\text { CIDI-AS } \\
(\mathrm{n}=391)\end{array}$ & $\begin{array}{l}6.00 \\
(10.00)\end{array}$ & $0.20(0.07)$ & $0.85(0.01)$ & $0.19(0.07)$ & $0.28(-0.06)$ & $0.38(-0.05)$ \\
\hline
\end{tabular}

ACC, accuracy; Ag, agency performance; BDI, Beck Depression Index; CIDI-AS, Anxiety Screening from the Composite International Diagnostic Interview; DAWBA-GA, Generalized Anxiety Scale from The Development and Well-Being Assessment Interview; FU2, IMAGEN second follow up assessment acquired at 19 years; GHSL, global human settlement layer; IRI, Interpersonal Reactivity Index; NDVI, normalized difference vegetation index; PT, perspective-taking; RSQ, ruminating scale questionnaire; RT, reaction time; SA, state anxiety; TA, trait anxiety. ${ }^{\#}$ Statistics are shown as median (quantile interval). *Spearman correlations are used to test the correlations between individual satellite measures and behaviors (except for DAWBA-GA) controlling for confounding covariates, which is shown as correlation P value (rho value). In the DAWBA-GA, logistic regression is used to test the correlations between individual satellite matures and anxiety, which is shown as $P$ value ( $\beta$ value). In CHIMGEN, the significant results after Bonferroni $P_{C}<0.05$ (uncorrected $P<0.05 / 2 / 21=1.19 \times 10^{-3}$ ) are in bold and italic; In IMAGEN, the significant results after Bonferroni $P C<0.05$ (uncorrected $P<0.05 / 5=0.01$ ) are in bold and italic.

\section{References}

1. Prince, M., et al. No health without mental health. Lancet 370, 859-877 (2007).

2. van Os, J., Kenis, G. \& Rutten, B.P. The environment and schizophrenia. Nature 468, 203 (2010).

3. Bick, J. \& Nelson, C.A. Early adverse experiences and the developing brain. Neuropsychopharmacology 41, 177 (2016). 
4. Castaldelli-Maia, J. \& McCay, L. Dinesh Bhugra, Antonio Ventriglio. Urban Mental Health (Oxford Cultural Psychiatry series), 354 (2019).

5. Vlahov, D. \& Galea, S. Urbanization, urbanicity, and health. J Urban Health 79, S1-S12 (2002).

6. Heilig, G.K. World urbanization prospects: the 2011 revision. United Nations, Department of Economic and Social Affairs (DESA), Population Division, Population Estimates and Projections Section, New York 14(2012).

7. Nations, U. 2018 revision of world urbanization prospects. (United Nations New York, 2018).

8. Evans, G.W. The built environment and mental health. J Urban Health 80, 536-555 (2003).

9. Melchiorri, M., et al. Unveiling 25 Years of Planetary Urbanization with Remote Sensing: Perspectives from the Global Human Settlement Layer. Remote Sens 10, 768 (2018).

10. Seltenrich, N. Remote-sensing applications for environmental health research. Environ Health Perspect 122, A268-275 (2014).

11. Xu, Q., et al. CHIMGEN: a Chinese imaging genetics cohort to enhance cross-ethnic and cross-geographic brain research. Mol Psychiatry, 1-13 (2019).

12. Schumann, G., et al. The IMAGEN study: reinforcement-related behaviour in normal brain function and psychopathology. Mol Psychiatry 15, 1128-1139 (2010).

13. Yeo, B.T., et al. The organization of the human cerebral cortex estimated by intrinsic functional connectivity. J Neurophysiol $106,1125-1165$ (2011).

14. Greicius, M.D., Krasnow, B., Reiss, A.L. \& Menon, V. Functional connectivity in the resting brain: a network analysis of the default mode hypothesis. Proc Natl Acad Sci U S A 100, 253-258 (2003).

15. Seeley, W.W., et al. Dissociable intrinsic connectivity networks for salience processing and executive control. J Neurosci 27, 2349-2356 (2007).

16. Lamm, C., Batson, C.D. \& Decety, J. The neural substrate of human empathy: effects of perspective-taking and cognitive appraisal. J Cogn Neurosci 19, 42-58 (2007).

17. Peen, J., Schoevers, R.A., Beekman, A.T. \& Dekker, J. The current status of urban-rural differences in psychiatric disorders. Acta Psychiatr Scand 121, 84-93 (2010).

18. Weiser, M., et al. Social and cognitive functioning, urbanicity and risk for schizophrenia. Br J Psychiatry 191, 320-324 (2007).

19. Hiser, J. \& Koenigs, M. The multifaceted role of the ventromedial prefrontal cortex in emotion, decision making, social cognition, and psychopathology. Biol Psychiatry 83, 638-647 (2018).

20. Henckens, M.J., et al. Stress-induced alterations in large-scale functional networks of the rodent brain. Neuroimage 105, 312-322 (2015).

21. Pujol, J., et al. Traffic pollution exposure is associated with altered brain connectivity in school children. Neuroimage 129, 175-184 (2016).

22. Haddad, L., et al. Brain structure correlates of urban upbringing, an environmental risk factor for schizophrenia. Schizophr Bull 41, 115-122 (2015).

23. Schmahmann, J.D. \& Sherman, J.C. The cerebellar cognitive affective syndrome. Brain 121 (Pt 4), 561-579 (1998).

24. Bambico, F.R., et al. High frequency stimulation of the anterior vermis modulates behavioural response to chronic stress: involvement of the prefrontal cortex and dorsal raphe? Neurobiol Dis 116, 166-178 (2018).

25. Carta, I., Chen, C.H., Schott, A.L., Dorizan, S. \& Khodakhah, K. Cerebellar modulation of the reward circuitry and social behavior. Science 363(2019).

26. Middleton, F.A. \& Strick, P.L. Cerebellar projections to the prefrontal cortex of the primate. J Neurosci 21, $700-712$ (2001).

27. Shaw, P., et al. Neurodevelopmental trajectories of the human cerebral cortex. J Neurosci 28, 3586-3594 (2008).

28. Lenroot, R.K. \& Giedd, J.N. Brain development in children and adolescents: insights from anatomical magnetic resonance imaging. Neurosci Biobehav Rev 30, 718-729 (2006).

Page $13 / 20$ 
29. Tiemeier, H., et al. Cerebellum development during childhood and adolescence: a longitudinal morphometric MRI study. Neuroimage 49, 63-70 (2010).

30. Lederbogen, F., et al. City living and urban upbringing affect neural social stress processing in humans. Nature 474, 498-501 (2011).

31. Price, C., Dalman, C., Zammit, S. \& Kirkbride, J.B. Association of residential mobility over the life course with nonaffective psychosis in 1.4 million young people in Sweden. JAMA Psychiatry 75, 1128-1136 (2018).

32. Fuhrmann, D., Knoll, L.J. \& Blakemore, S.J. Adolescence as a Sensitive Period of Brain Development. Trends Cog Sci 19, 558-566 (2015).

33. Tang, Y., et al. Brain structure differences between Chinese and Caucasian cohorts: A comprehensive morphometry study. Hum Brain Mapp 39, 2147-2155 (2018).

34. Douglas Bremner, J.J.C. \& Targets, N.D.-D. Stress and brain atrophy. 5, 503-512 (2006).

35. Guxens, M., et al. Air pollution exposure during fetal life, brain morphology, and cognitive function in school-age children. Biol Psychiatry 84, 295-303 (2018).

36. Khan, A., et al. Environmental pollution is associated with increased risk of psychiatric disorders in the US and Denmark. PLoS Bio/ 17, e3000353 (2019).

37. Weiser, M., et al. Social and cognitive functioning, urbanicity and risk for schizophrenia. Br J Psychiatry 191, 320-324 (2007).

38. Tyborowska, A., et al. Early-life and pubertal stress differentially modulate grey matter development in human adolescents. Sci Rep 8, 1-11 (2018).

39. Paus, T. Mapping brain maturation and cognitive development during adolescence. Trends Cog Sci 9, 60-68 (2005).

40. Callaghan, B.L. \& Tottenham, N. The stress acceleration hypothesis: Effects of early-life adversity on emotion circuits and behavior. Curr Opin Behav Sci 7, 76-81 (2016).

41. Fonken, L.K., et al. Air pollution impairs cognition, provokes depressive-like behaviors and alters hippocampal cytokine expression and morphology. Mol Psychiatry 16, 987-995 (2011).

42. Elvidge, C.D., et al. Radiance calibration of DMSP-OLS low-light imaging data of human settlements. Remote Sens Environ 68, 77-88 (1999).

43. Liu, Z., et al. Extracting the dynamics of urban expansion in China using DMSP-OLS nighttime light data from 1992 to 2008. Landsc Urban Plan 106, 62-72 (2012).

44. Ma, T., Zhou, C., Pei, T., Haynie, S. \& Fan, J.J.R.S.o.E. Quantitative estimation of urbanization dynamics using time series of DMSP/OLS nighttime light data: A comparative case study from China's cities. Remote Sens Environ 124, $99-107$ (2012).

45. Ghosh, T., et al. Shedding light on the global distribution of economic activity. Open Geogr J 3(2010).

46. Helbich, M.J.I.j.o.e.r. \& health, p. Spatiotemporal contextual uncertainties in green space exposure measures: exploring a time series of the normalized difference vegetation indices. Int J Environ Res Public Health 16, 852 (2019).

\section{Figures}



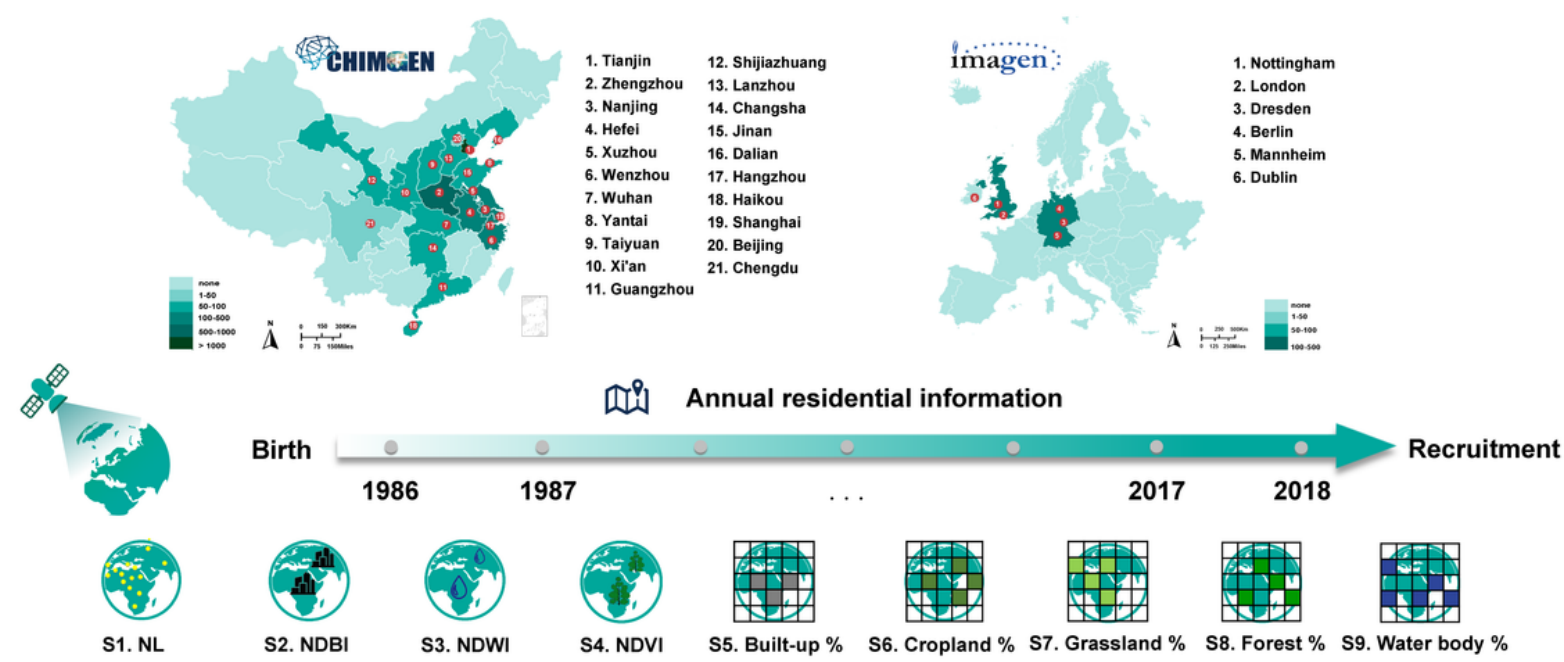

b

UrbanSat from 10-fold cross validation of CFA model

Correlation with population density

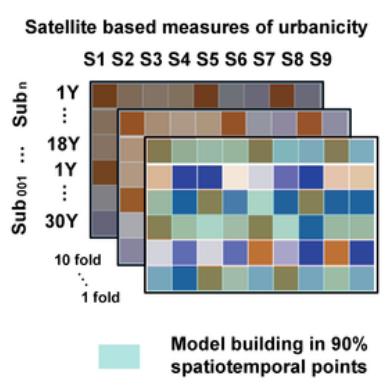

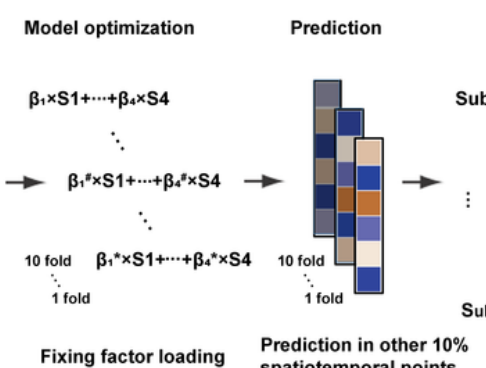

Fixing factor loading $\quad \begin{aligned} & \text { Prediction in other } \\ & \text { spatiotemporal points }\end{aligned}$

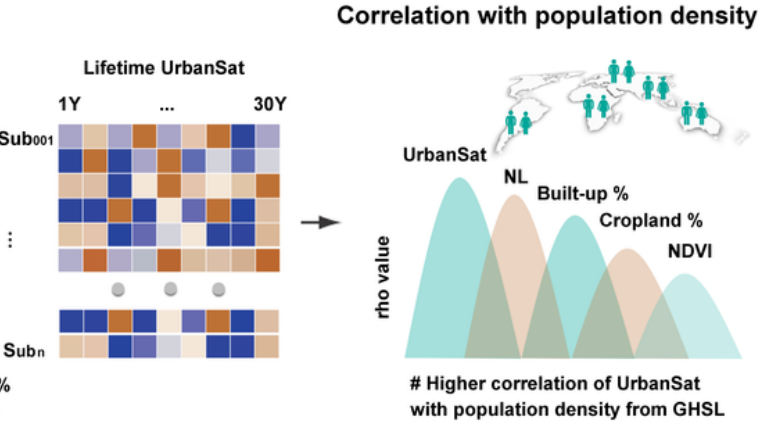

C

Cumulitive effect of UrbanSat on brain and behaviors d

Susceptibility periods of lifetime UrbanSat
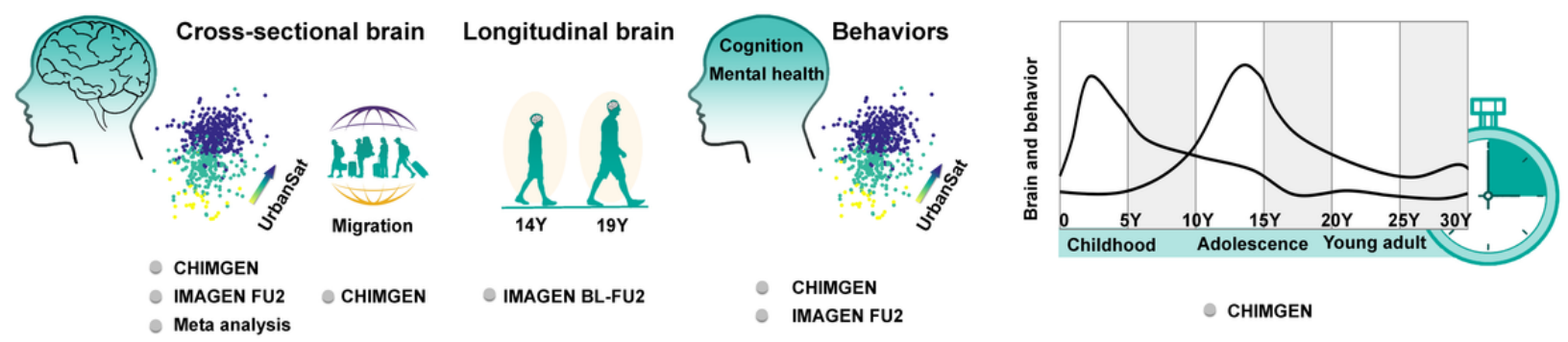

CHIMGEN

Figure 1

Schematic summary of study design. a. Top: The geographic distribution of recruitment sites and number of participants collected in each city of China and in each country of Europe. Five sites in Tianjin, two sites each in Zhengzhou, Nanjing, Hefei and Wenzhou; one site in the remaining cities. Bottom: Lifetime residential geopositions of each participant were collected from 1986 to 2018. Based on the individual geoposition data, the annual values of nine satellite-measures of urbanicity are obtained for each participant, including the night-time light (NL), normalized difference built-up index (NDBI), normalized difference water index (NDWI), normalized difference vegetation index (NDVI) and five measures derived from land cover mapping (Built-up\%, cropland\%, grassland\%, forest $\%$ and water body\%). b. Ten-fold cross validation of confirmatory factor analysis (CFA) stratified by spatiotemporality was applied to predict annual UrbanSat score for each participant. The optimized CFA model includes NL, built-up\%, cropland\% and NDVI. The mean UrbanSat scores before 18 years showed higher correlation with ground level population-density from global human settlement layers (GHSL) than any individual satellite-measures both in CHIMGEN and IMAGEN-FU2. c. Investigation of the cumulative effects of UrbanSat on brain and behavior. d. Identification of susceptibility periods of lifetime UrbanSat on brain and behavior using distributed lag models. S, satellite-measures of urbanicity; Sub, subjects; Y, years old. Note: The designations employed and the presentation of the material on this map do not imply the 
expression of any opinion whatsoever on the part of Research Square concerning the legal status of any country, territory, city or area or of its authorities, or concerning the delimitation of its frontiers or boundaries. This map has been provided by the authors.
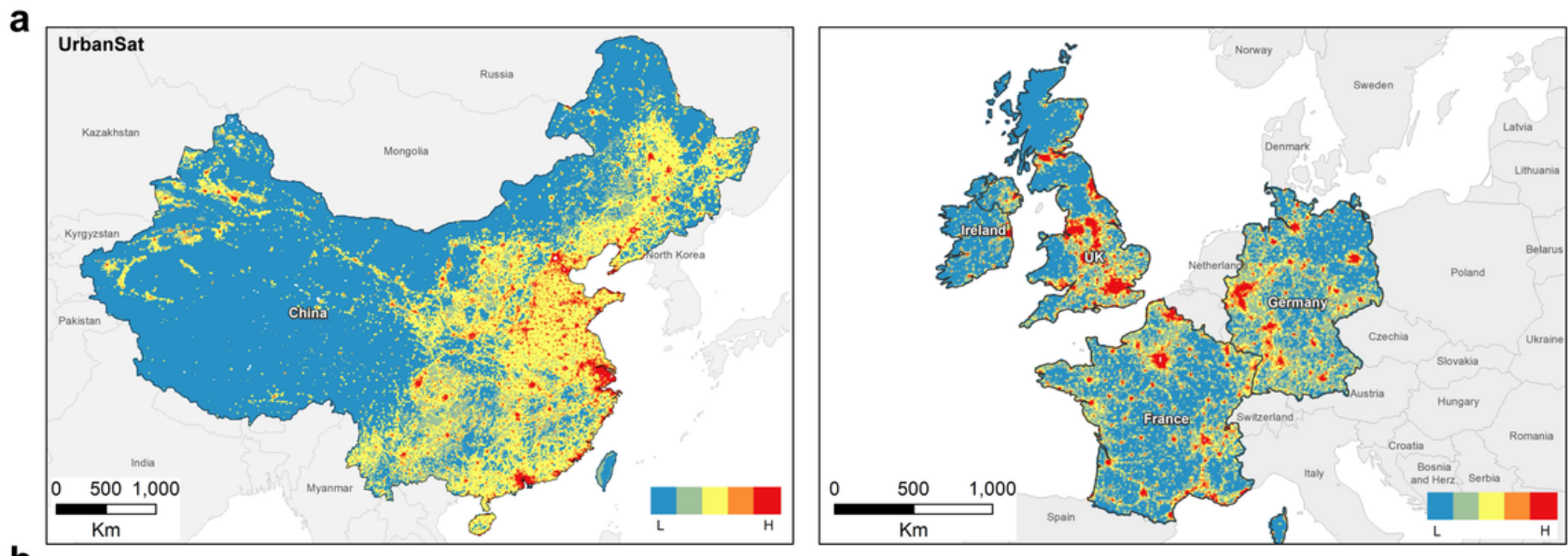

b
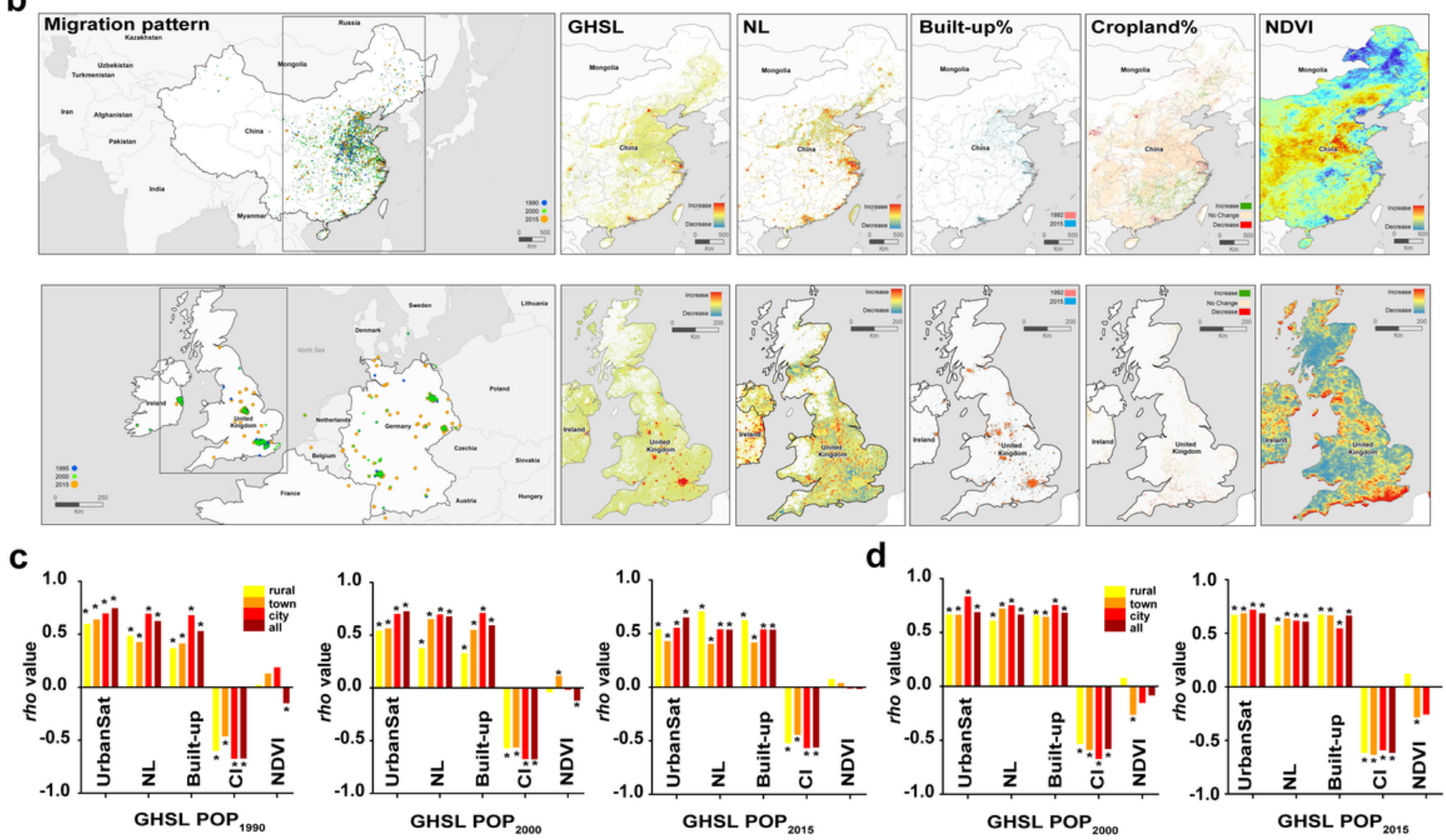

\section{Figure 2}

Maps of satellite-based measures of urbanicity in China and Europe. a. UrbanSat maps of China (left) and Europe (right) in 2013. b. Maps showing temporal changes of satellite-measures of urbanicity during available years in China (top) and Europe (bottom). Left: Migration patterns of 3306 participants in CHIMGEN and 561 participants in IMAGEN-FU2. The blue, green and orange dots represent geographic locations of participants in the year of 1990, 2000 and 2015, respectively. The box on the map represents the extent of zoom-in maps of each satellite-measure of urbanicity in the right columns. Right: Changes of GHSL population-density between 1990 and 2015, NL between 1992 and 2013, built-up\% and cropland\% between 1992 and 2015, NDVI between 1981 and 2017 in zoom-in part of China and Europe. Note that different time ranges are available for different satellite registrations. $\mathrm{c}$ and d. Correlations of UrbanSat and individual satellite-measures with population-density in 3306 CHIMGEN (c) and 561 IMAGEN-FU2 (d) participants. Columns demonstrate rho value (y-axis) of the UrbanSat and individual (NL, built-up, cropland and NDVI) satellite-measures (x-axis) with GHSL population-density in rural (yellow), town (orange), city 


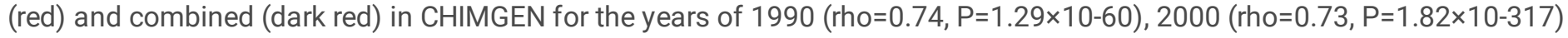
and 2015 (rho=0.65, $P=3.73 \times 10-244$ ), and in IMAGEN for the years of 2000 (rho=0.69, $P=1.75 \times 10-55$ ) and 2015 (rho=0.69, $\mathrm{P}=3.40 \times 10-39)$. Cl, cropland; GHSL POP, population-density of global human settlement layers; NDVI, normalized difference vegetation index; NL, night-time light. *Significant correlations of satellite-measures of urbanicity with population-density using Spearman correlation. Note: The designations employed and the presentation of the material on this map do not imply the expression of any opinion whatsoever on the part of Research Square concerning the legal status of any country, territory, city or area or of its authorities, or concerning the delimitation of its frontiers or boundaries. This map has been provided by the authors.

a
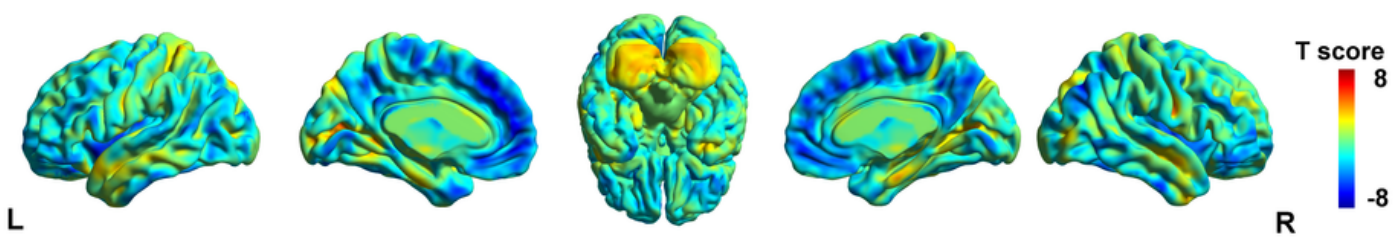

b
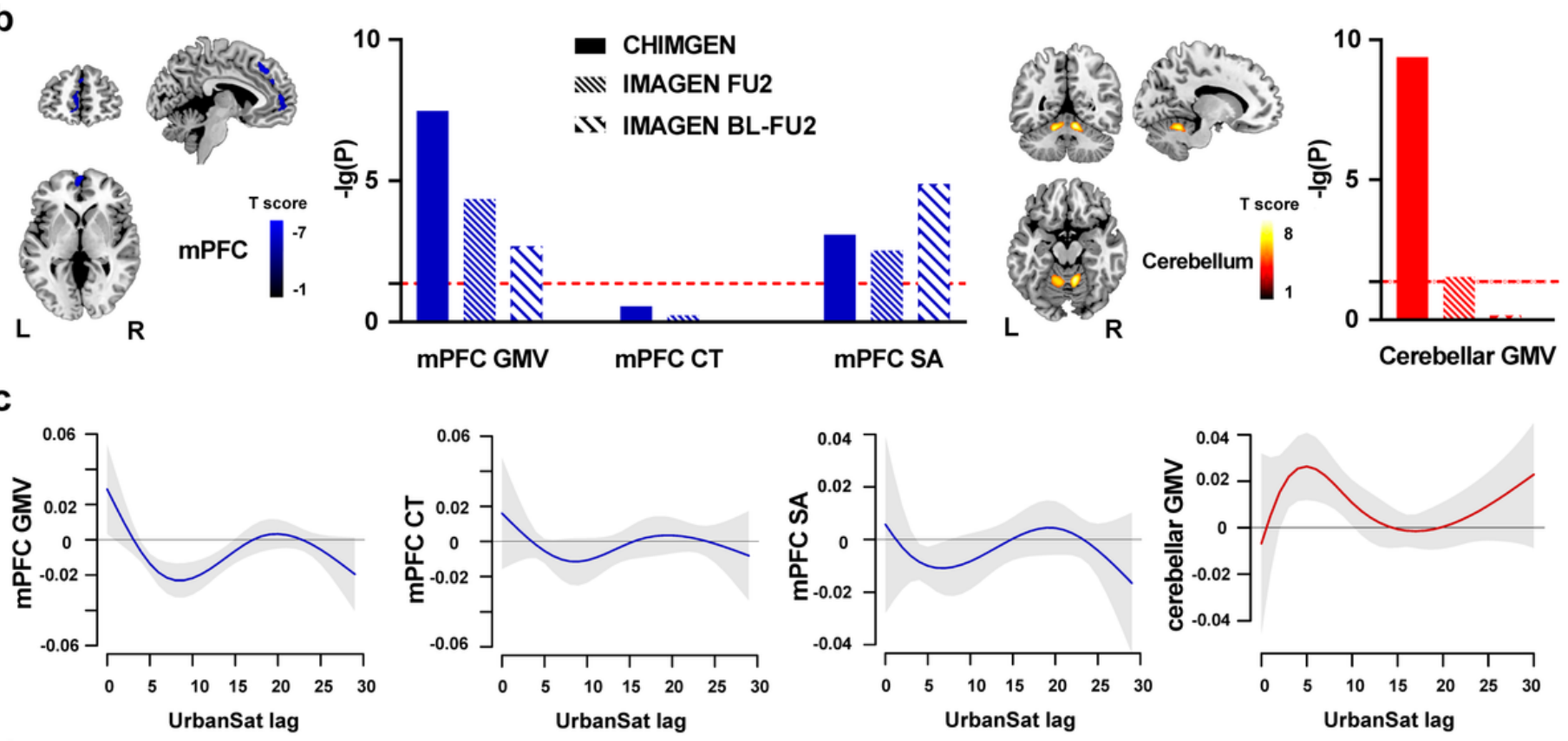

C
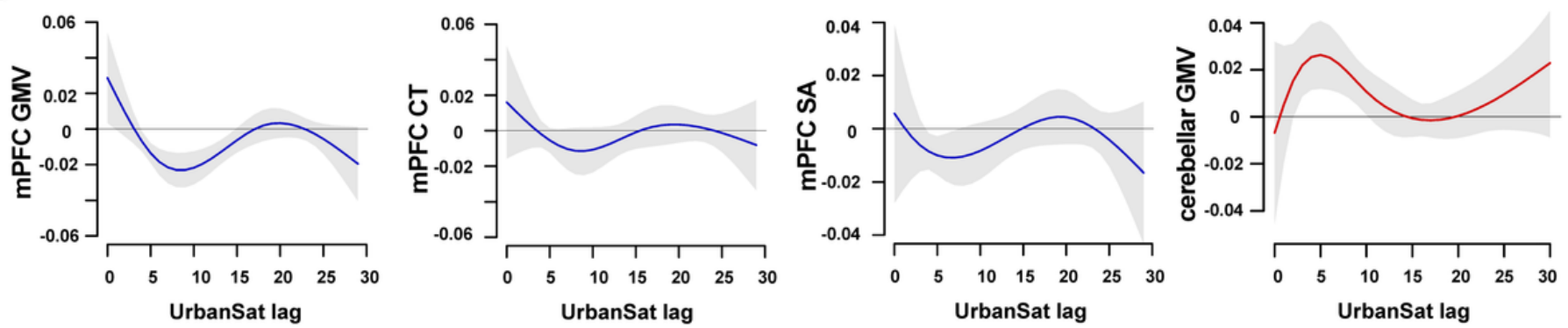

\section{d}

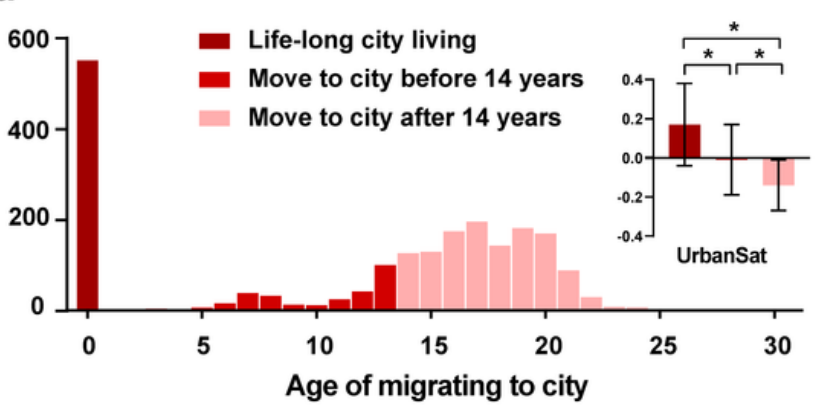

e
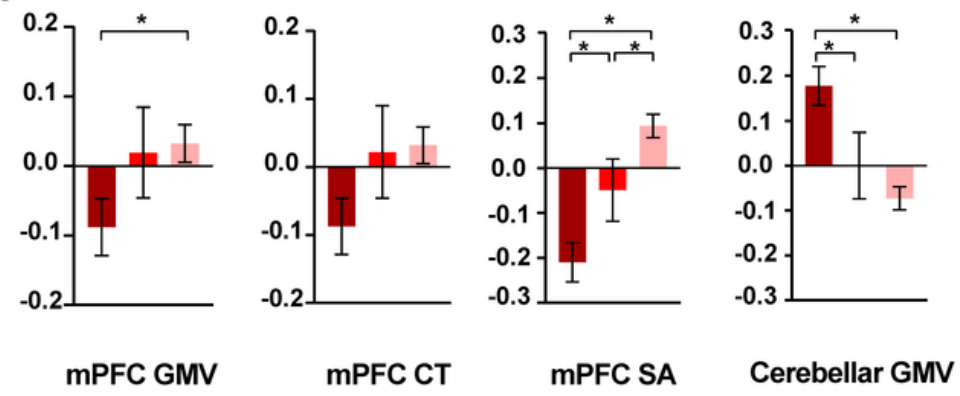

Figure 3

Correlations of UrbanSat with brain structure. a. Uncorrected statistical maps in the voxel-wise multiple regression of mean UrbanSat before 18 years with brain GMV under parametric testing in CHIMGEN ( $n=2176)$. b. In CHIMGEN, UrbanSat is negatively (blue) correlated with mPFC GMV (left) and positively correlated with cerebellar GMV (right) (FWE Pc<0.05). The correlation of UrbanSat with $\mathrm{mPFC} \mathrm{GMV}$ is driven by SA rather than CT and these correlations are replicated in IMAGEN-FU2 $(n=415)$; UrbanSat is correlated with brain volumetric change in the mPFC $(n=340)$ between 14 and 19 years in IMAGEN BL-FU2, 
which is driven by the change of SA rather than CT $(n=325)$. The dashed red lines indicate the threshold of $P=0.05$. $c$.

Susceptibility-periods analysis of brain structure using DLM. In CHIMGEN, we observed a negative association of UrbanSat with mPFC volume during childhood and adolescence (age 4-15 years) and mPFC SA during childhood (age 5-7 years old) as well as a positive association with cerebellar volume during childhood (age 1-10 years). The y-axis represents the changes of brain structural features associated with an increase of interquartile range of UrbanSat; the x-axis is UrbanSat lag in ages. Grey areas indicate $95 \%$ confidence intervals $(\mathrm{Cl})$. A susceptibility window is identified for the ages where the estimated pointwise $95 \% \mathrm{Cl}$ (shaded area) does not include zero. d. Lower left: Numbers of participants migrating to city at different ages. Upper right: UrbanSat is highest in participants with life-long city living $(n=562)$ (dark red), medium in participants moving to city before 14 years $(n=229)$ (red) and lowest in participants moving to city after 14 years $(n=1385)$ (light red) $(P=5.96 \times 10-256)$. e. The participants who were born in the city or migrated to an urban environment at an earlier age showed smaller mPFC-GMV $(P=0.040)$ and mPFC-SA $(P=7.28 \times 10-9)$ as well as greater cerebellar-GMV $(P=5.00 \times 10-5)$ than those with later exposure. $B L$, IMAGEN baseline assessment acquired at 14 years old; BL-FU2, IMAGEN BL-FU2 measures brain structural changes rate between BL of 14 years and FU2 of 19 years; CT, cortical thickness; DLM, distributed lag model; FU2, IMAGEN second follow up assessment acquired at 19 years old; GMV, gray matter volume; L, left; mPFC, medial prefrontal cortex; R, right; SA, surface area. $* \mathrm{P}<0.05$.
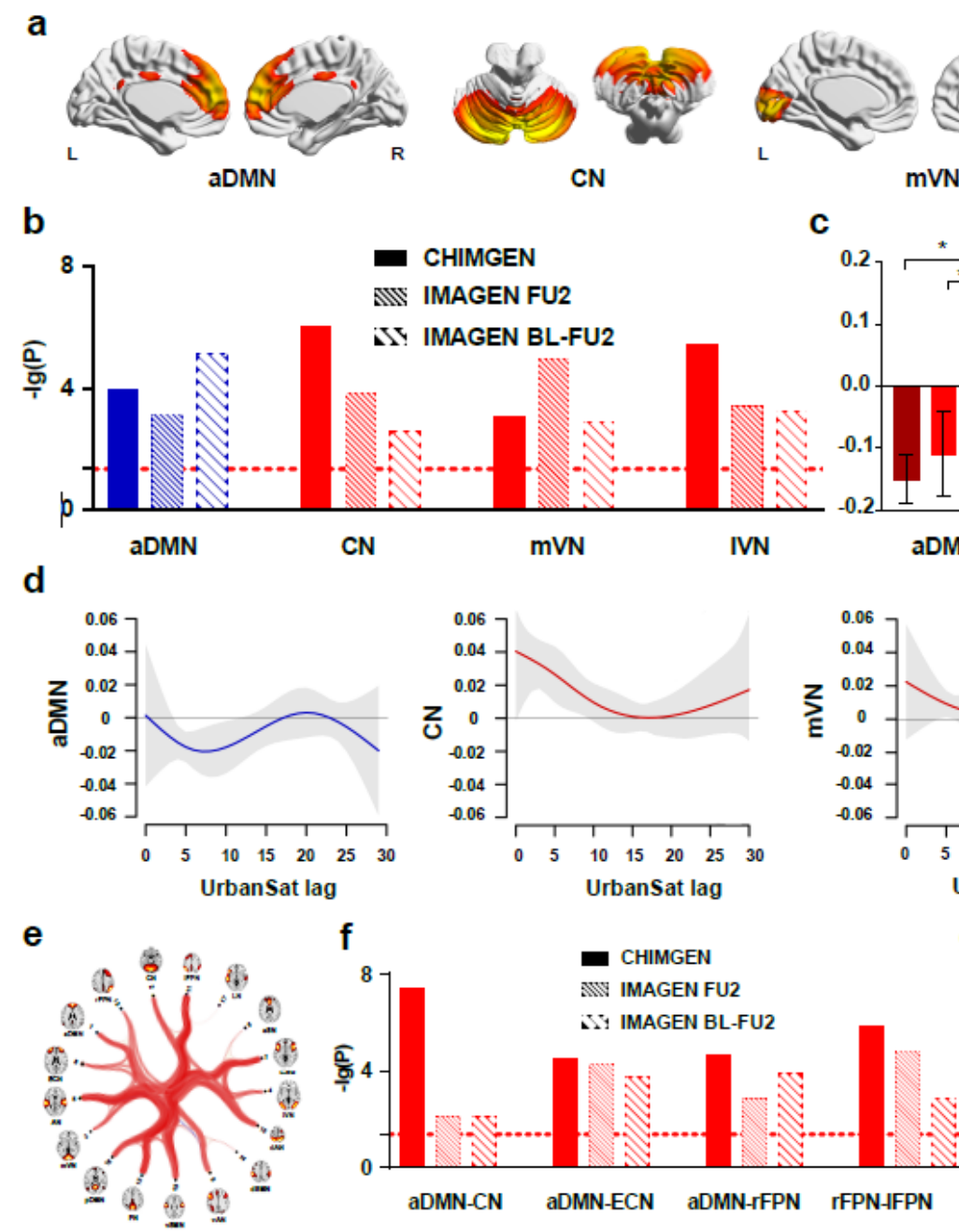

CHIMGEN IMAGEN FU2 IMAGEN BL-FU2 CN

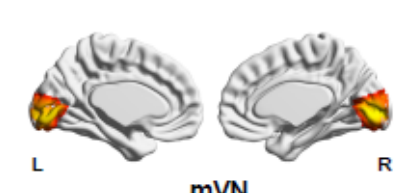

$\mathrm{mVN}$

C

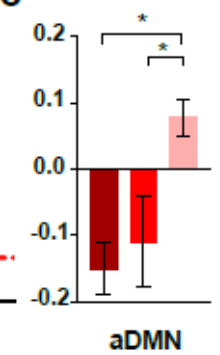

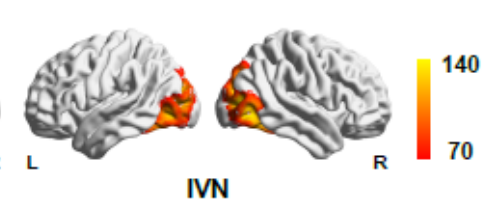

IVN

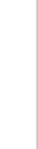
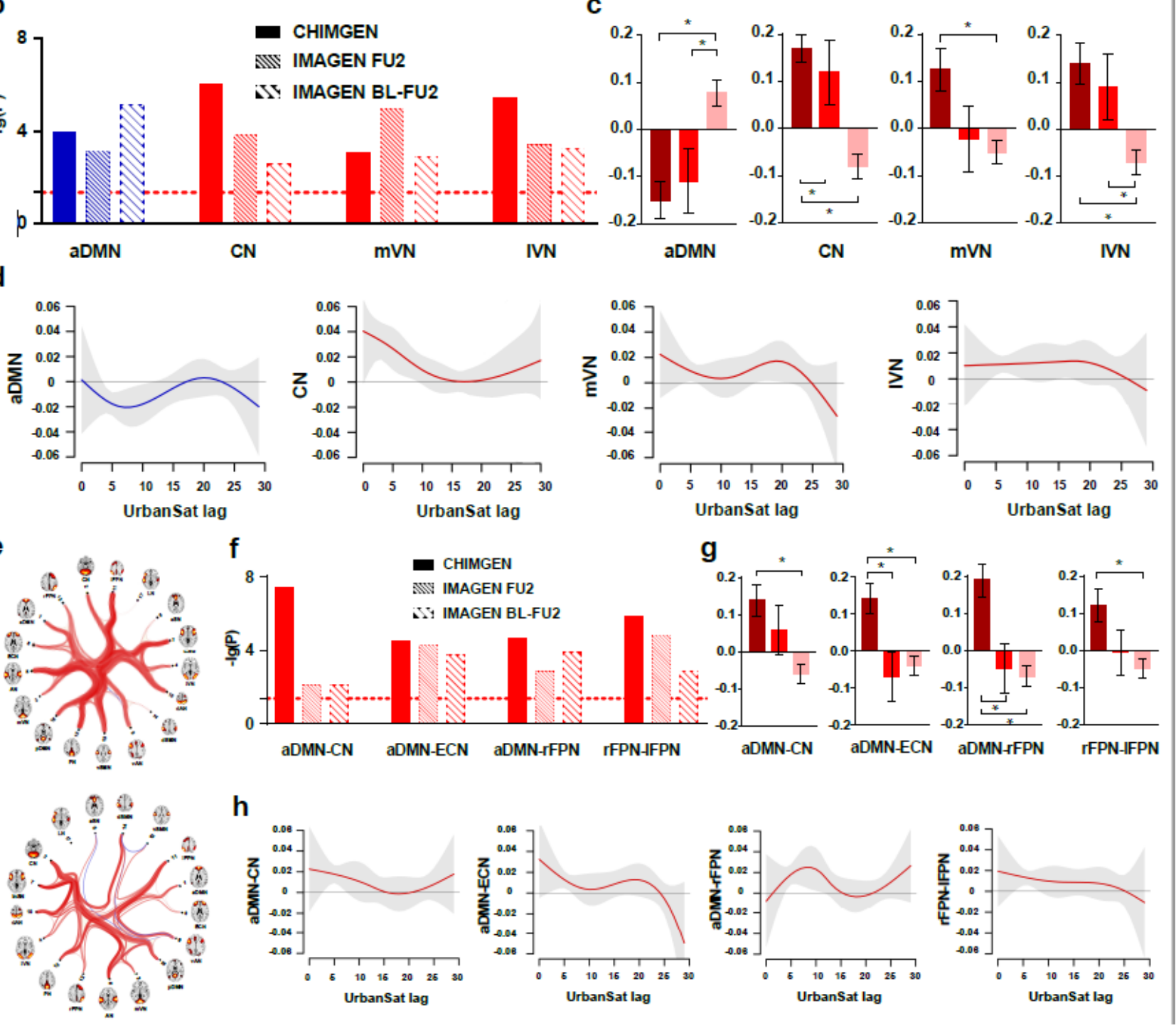


\section{Figure 4}

Correlations of UrbanSat with within-network (WNFC) and between-network (BNFC) functional connectivity. a. Voxel-wise multiple regression analysis controlling for confounders identified four WNFCs (aDMN, CN, mVN and IVN) correlated with mean UrbanSat before 18 years in CHIMGEN $(n=2156)$. b. UrbanSat is negatively (blue) correlated with WNFC in the mPFC of the aDMN, positively correlated (red) with WNFC in the CV of the CN and left LG of the mVN and IVN (FWE Pc<0.05). These correlations are replicated in IMAGEN-FU2 $(n=351)$. UrbanSat is correlated with WNFCs change rate/year in these four WNFCs between 14 and 19 years in IMAGEN ( $n=83)$. c. The lifelong city dwellers $(n=559)$ (dark red) and earlier migrants $(n=222)(r e d)$ showed greater WNFCs in the CN, mVN and IVN but smaller WNFC in the aDMN than later migrants ( $n=1375)$ (light red); $d$. Susceptibility period analysis of WNFCs using DLM. In CHIMGEN, we observed a significant negative association of UrbanSat with WNFC in aDMN during childhood and adolescence (ages 4-12 years), a positive association with WNFC in CN during childhood (ages 0-9 years) as well as with WNFC in IVN during childhood (ages 5-9 years). No significant susceptibility-periods are observed in the association of UrbanSat with WNFC in $\mathrm{mVN}$. The y-axis represents the changes in WNFCs associated with an increase of interquartile range of UrbanSat; the $x$-axis is UrbanSat lag in ages. Grey areas indicate $95 \%$ confidence intervals (Cl). A susceptibility window is identified for the ages where the estimated pointwise $95 \% \mathrm{Cl}$ (shaded area) does not include zero. e. There are 49 BNFCs correlating with UrbanSat and 45 BNFCs correlating with night-time light in CHIMGEN $(P c<0.05$, 10,000 permutations). f. Correlations of UrbanSat with BNFCs of aDMN-CN, aDMN-ECN, aDMN-rFPN and rFPN-IFPN are replicated in IMAGEN-FU2; UrbanSat is also correlated with these four BNFCs changes rate/year between 14 and 19 years in IMAGEN BL-FU2. g. The lifelong city dwellers (dark red) and earlier migrants (red) showed greater BNFCs than later migrants (light red); h. Susceptibility-periods analysis of BNFCs using DLM. In CHIMGEN, we observed a significant positive association of UrbanSat with BNFCs of aDMN-CN (ages 3-7 years), aDMN-ECN (ages 3-6 years), aDMN-rFPN (ages 4-10 years) and rFPNIFPN (ages 4-9 years) during childhood periods. aDMN, anterior default mode network; CN, cerebellar network; CV, cerebellar vermis; DLM, distributed lag models; ECN, executive control network; L, left; LG, lingual gyrus; IFPN, left frontoparietal network; IVN, lateral visual network; mPFC, medial prefrontal cortex; $\mathrm{mVN}$, medial visual network; R, right; rFPN, right frontoparietal network. 
a
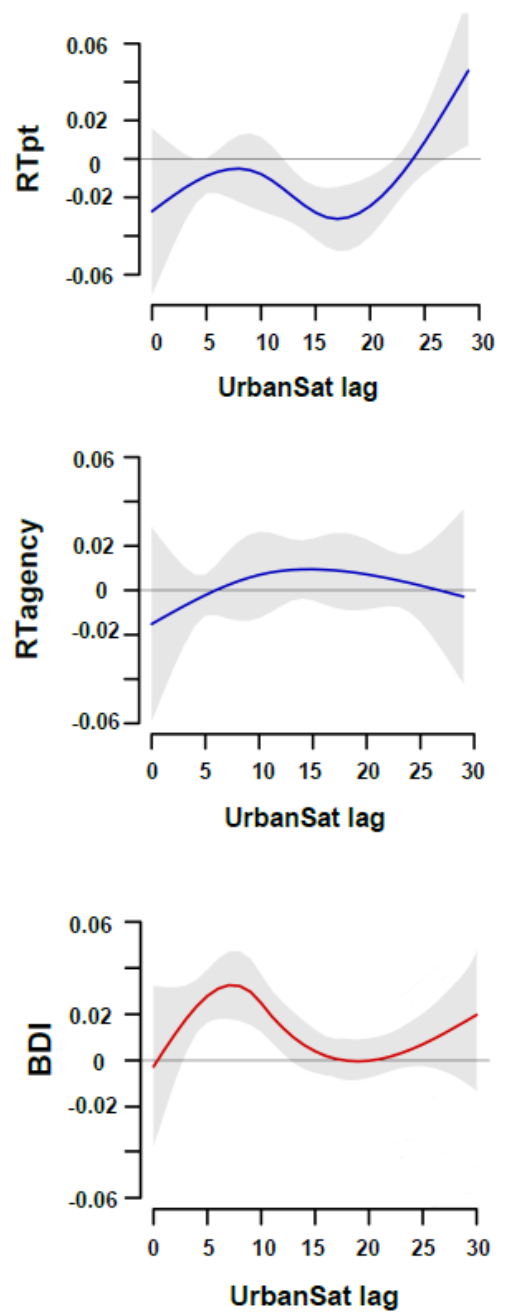

b
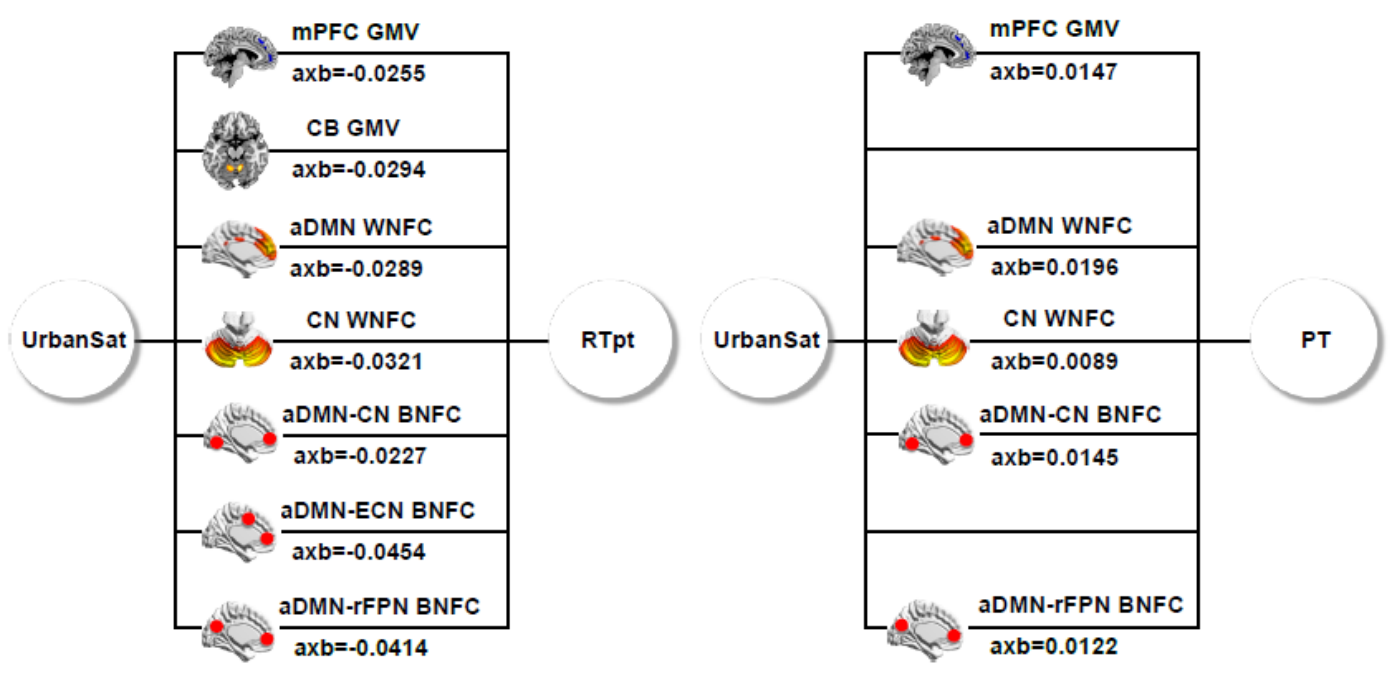

C

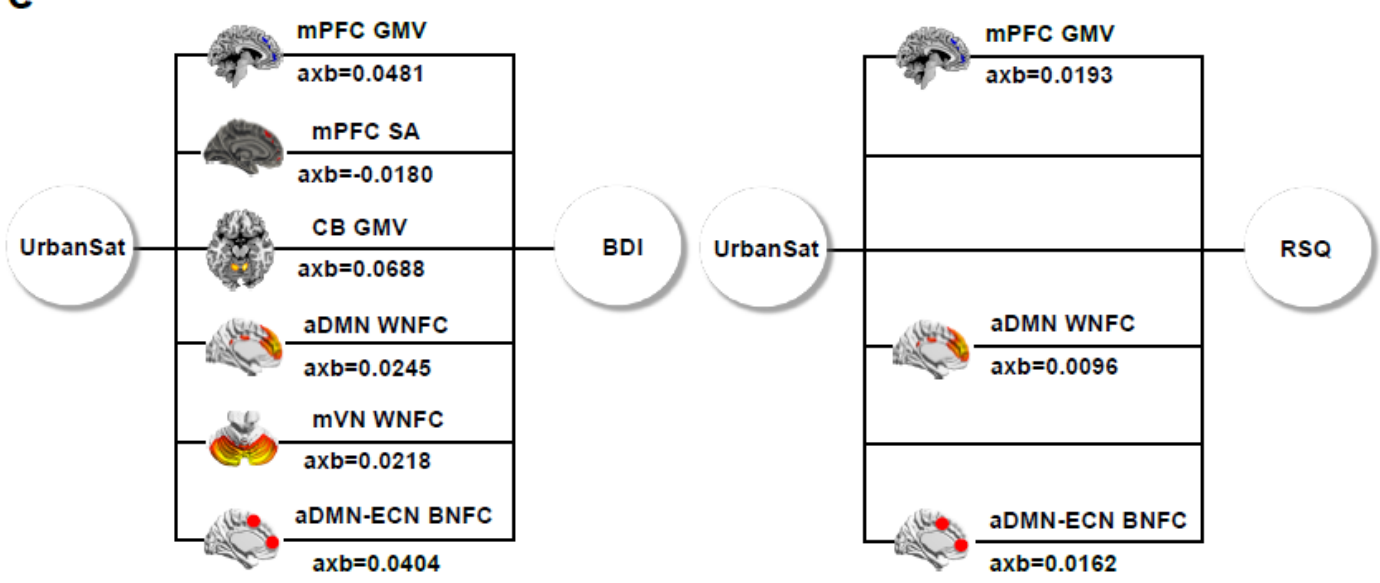

Figure 5

Susceptibility-periods of behavior using DLM and multiple mediation of UrbanSat-brain-behavior. a. UrbanSat is negatively correlated with reaction-time for perspective-taking (top) from age 12 to 22 years but not with that for agency (medium) in CHIMGEN ( $n=2148)$. UrbanSat is positively correlated with depression-symptoms (BDI) from age 3 to 12 years in CHIMGEN ( $n=2170$ ) (bottom). b. In CHIMGEN, the correlation of UrbanSat with perspective-taking performance is mediated by mPFC and cerebellar GMVs, WNFCs in aDMN and CN, as well as BNFCs of aDMN-CN, aDMN-ECN and aDMN-rFPN (left); These mediation effects are replicated in IMAGEN-FU2 except for cerebellar GMV and BNFC of aDMN-ECN (right). c. In CHIMGEN, the correlation between UrbanSat and BDI is mediated by MPFC GMV and SA, cerebellar GMV, WNFCs in aDMN and mVN as well as BNFC of aDMN-ECN (left); The mediation effects of the MPFC GMV, WNFC in aDMN and BNFC of aDMN-ECN are replicated in IMAGENFU2 (right). aDMN, anterior default mode network; BDI, Beck depression index; CN, cerebellar network; DLM, distributed lag models; ECN, executive-control-network; IFPN/rFPN, left or right frontoparietal-network; mPFC, medial prefrontal cortex; PT, perspective-taking measured by interpersonal reactivity index (IRI); RSQ, ruminating scale questionnaire; RTpt and RTagency, reaction-time for perspective-taking and agency.

\section{Supplementary Files}

This is a list of supplementary files associated with this preprint. Click to download.

- 20201121xujiayuanNMsupplaintext.doc 